\title{
An impedance-based control architecture for multi-robot cooperative dual-arm mobile manipulation
}

\author{
Sebastian Erhart, Dominik Sieber and Sandra Hirche
}

\begin{abstract}
Cooperative manipulation in robotic teams likely results in an increased manipulation performance due to complementary sensing and actuation capabilities or increased redundancy. However, a precise coordination of the involved manipulators is required in order to avoid undesired stress on the manipulated object. Extending the workspace of the robots by means of mobile platforms greatly enlarges the potential task spectrum but simultaneously poses new challenges for example in terms of increased kinematic errors. In this paper we show how kinematic errors in the closed kinematic chain originating from uncertainties in the geometry of object and manipulators limit the cooperative task performance. We extend an impedance-based coordination control scheme towards mobile multi-robot manipulation to limit undesired internal forces in the presence of kinematic uncertainties. Furthermore, we employ a task-space decoupling approach to reduce the impact of disturbances at the mobile platforms on the end effectors. The presented control scheme for cooperative, mobile dualarm manipulation is applicable in real-time and suitable for a team of heterogeneous manipulators. We evaluate the presented architecture by means of a large-scale experiment with four 7DoF manipulators on two mobile platforms.
\end{abstract}

\section{INTRODUCTION}

Cooperation of robotic manipulators allows to accomplish tasks which would exceed the capacity of a single manipulator. Depending on the specific requirements, distributed sensing and actuation capabilities can be merged to increase the manipulation performance or the available redundancy. A successful manipulation task is characterized by moving an object accurately and without undesired stress on the object along a desired trajectory. The motion of the manipulators needs to be coordinated in order to reach the object's goal pose and limit undesired stress on the object. Mobile manipulation is of particular interest since it increases significantly the workspace of the manipulators and therewith extends manipulation tasks to large-scale environments. A potential cooperative manipulation task is depicted in Fig. 1.

Cooperative manipulation schemes aim to achieve a global control goal by orchestrating a group of manipulators relying on local sensor information. Each manipulator in turn interacts with the object by means of some local control action. Of particular significance for mobile cooperative manipulation is the uncertainty in local pose sensing arising at object and manipulator level, which leads to notable differences in the relative manipulator displacements. Adding now mobility those uncertainties tend to accumulate when manipulating cooperatively over longer distances and inject a significant

All authors are with the Institute for Information-oriented Control, Technische Universität München, D-80290 Munich, Germany. \{erhart, dominik.sieber, hirche\}@tum.de disturbance into the system. In the worst case, significant stress is exerted by the manipulators risking a severe damage of the object.

Impedance-based control schemes for single manipulators [1] and cooperating manipulators [2], [3] prove useful for dynamic interaction tasks. A decentralized strategy for cooperating single arm mobile manipulators is presented [4] in which uncertain kinematic grasp parameters entail a complete recomputation of the manipulators' task specification. Decoupling of locomotion and manipulation for cooperating $3 \mathrm{DoF}$ manipulators is presented in [5] which relaxes the workspace constraints due to odometry errors. A dynamic decoupling strategy of mobile platform and dual-arm manipulator for a human-robot manipulation task is illustrated in [6], however, without accounting for kinematic errors. Identification of uncertain kinematic parameters of an individual manipulator and the closed kinematic chain built by two manipulators is addressed in [7]-[9]. The authors of [10] present a cooperative control scheme for mobile manipulators without considering kinematic uncertainties. Generally the task specification in terms of desired interaction forces will only be met when the kinematics are accurately known and incorporated into the control law. Currently there is no cooperative control scheme for anthropomorphic mobile manipulators which effectively copes with the challenge of task coordination under uncertain kinematic parameters.

In this paper we propose a control scheme for cooperative, dual-arm mobile manipulation. We present an extensive formulation of kinematic uncertainties and emphasize their impact on the overall system performance. A consistent modeling of those uncertainties at the object and the ma-

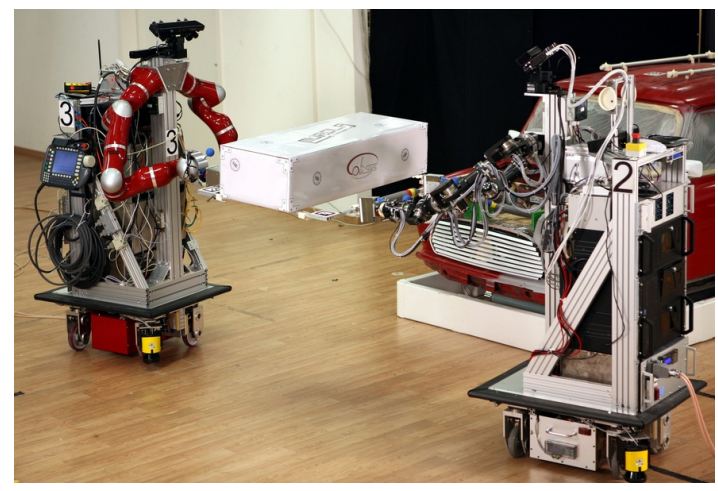

Fig. 1. Anthropomorphic mobile robots transporting an object cooperatively 
nipulator level is presented and evaluated by means of an internal force analysis. The coordination problem of the emerging kinematic tree structure built by a mobile platform and two anthropomorphic manipulators is resolved by a decoupling technique in task-space. The presented scheme is applicable to a team of heterogeneous manipulators in realtime for the coordination of the object motion. The impact of accumulated uncertainty on the manipulation performance is demonstrated in an experimental study with four $7 \mathrm{DoF}$ manipulators on two mobile platforms. The results illustrate the robustness of the presented architecture against biased kinematic parameters.

The remainder of this paper is organized as follows. Section II introduces the model of the manipulators, the object and the interaction forces. Section III describes the cooperative control scheme. In Section IV we present experimental data and discuss the results.

\section{Model of COOPERATIVE MANipUlation With KINEMATIC UNCERTAINTIES}

We consider the problem of multiple mobile manipulators rigidly grasping an object and thus forming a closed kinematic chain. The mobile manipulator setup is depicted in Fig. 2.

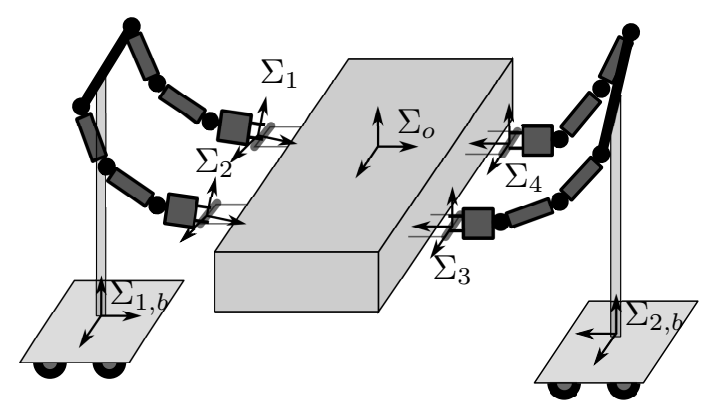

Fig. 2. Coordinate frames for mobile robots and object

For the $i$-th manipulator, position and orientation of the end effector frame $\Sigma_{i}$ is expressed in the base coordinate system $\Sigma_{k, b}$ of the mobile system. The object pose is represented by the frame $\Sigma_{o}$ aligned to the principal axes of the object with its origin in the center of mass. A superscript ()$^{s}$ indicates a vector which is represented in the coordinate system of frame $\Sigma_{s}$. If not stated otherwise, vectors are represented in the appropriate base frame $\Sigma_{k, b}$ with $k \in\{1,2\}$.

\section{A. Object dynamics and grasp geometry}

The manipulated object is assumed to be rigid and of known mass $m_{o}$ and inertia $J_{o}$. Choosing the Cartesian position and orientation as the state variable $x \in S E(3)$, the translational and angular velocities $\dot{p} \in \mathbb{R}^{3}$ and $\omega \in \mathbb{R}^{3}$ define the rigid object twist

$$
\dot{x}=\left(\begin{array}{c}
\dot{p} \\
\omega
\end{array}\right) \in \mathbb{R}^{6} .
$$

The object's equation of motion expressed in the body-fixed frame $\Sigma_{o}$ is

$$
M_{o}^{o} \ddot{x}_{\mathrm{obj}}^{o}+C_{o}^{o}=f_{\mathrm{obj}}^{o}
$$

with

$$
M_{o}^{o}=\left[\begin{array}{cc}
m_{o} I_{3} & 0_{3} \\
0_{3} & J_{o}
\end{array}\right] \quad \text { and } \quad C_{o}^{o}=\left[\begin{array}{c}
-m_{o} g^{o} \\
\omega_{\mathrm{obj}}^{o} \times J_{o} \omega_{\mathrm{obj}}^{o}
\end{array}\right] \text {, }
$$

where $\ddot{x}_{\text {obj }}^{o} \in \mathbb{R}^{6}$ denotes the object's linear and angular acceleration in the object coordinate frame and $f_{\text {obj }}^{o} \in \mathbb{R}^{6}$ represents the applied wrench to the object. $I_{3} \in \mathbb{R}^{3 \times 3}$ and $0_{3} \in \mathbb{R}^{3 \times 3}$ are the identity matrix and the null matrix respectively. The matrix $C_{o}^{o} \in \mathbb{R}^{6 \times 3}$ accounts for gravity and Coriolis forces.

We assume that the desired trajectory $x_{\mathrm{obj}, d}^{o}(t) \in S E(3)$ of the object is collision-free and feasible for the robotic manipulators, obtained from high-level reasoning and an associated path planning algorithm. Based on a specific orientation representation it is always possible to compute the corresponding angular velocity, i.e. the desired object twist $\dot{x}_{\mathrm{obj}, d}^{o}(t)$ is well-defined. The relation between the object's velocity and the compatible motion of the $i$-th end effector is expressed by means of the twist transformation matrix [11]

$$
G_{i}\left(\hat{\rho}_{i}\right)=\left[\begin{array}{cc}
I_{3} & 0 \\
S\left(\hat{\rho}_{i}\right) & I_{3}
\end{array}\right],
$$

with the skew-symmetric cross-product matrix $S(.) \in \mathbb{R}^{3 \times 3}$ and the estimated grasp point location $\hat{\rho}_{i} \in \mathbb{R}^{3}$ of the $i$-th end effector. The estimated grasp point orientation is expressed by $\hat{\eta}_{i} \in S O(3)$ based on an appropriate orientation parameterization. The ideal grasp geometry is characterized by the parameters $\rho_{i}^{*} \in \mathbb{R}^{3}$ and $\eta_{i}^{*} \in S O(3)$. The transformation from frame $\Sigma_{o}$ to the particular end effector frame $\Sigma_{i}$ is expressed by the rotation matrix $R\left(\hat{\eta}_{i}\right) \in \mathbb{R}^{3 \times 3}$. For notational convenience the rotational transformation is combined into $R_{i}\left(\hat{\eta}_{i}\right)=\left[R\left(\hat{\eta}_{i}\right), 0_{3} ; 0_{3}, R\left(\hat{\eta}_{i}\right)\right]$ which yields

$$
\dot{x}_{i, d}=R_{i}\left(\hat{\eta}_{i}\right) G_{i}^{T}\left(\hat{\rho}_{i}\right) \dot{x}_{\mathrm{obj}, d}^{o}
$$

for the desired velocity transformation. Splitting up the matrices $R_{i}\left(\hat{\eta}_{i}\right)=R_{i}\left(\hat{\eta}_{i}^{*}\right)+\Delta R_{i}\left(\eta_{i}^{*}, \hat{\eta}_{i}\right) \quad$ and $G_{i}\left(\hat{\eta}_{i}\right)=G_{i}\left(\hat{\rho}_{i}^{*}\right)+\Delta G_{i}\left(\rho_{i}^{*}, \hat{\rho}_{i}\right)$ in terms of ideal and estimated grasp parameters allows rewriting of (5) as

$$
\dot{x}_{i, d}=R G_{i}^{*} \dot{x}_{\mathrm{obj}, d}^{o}+\Delta R G_{i} \dot{x}_{\mathrm{obj}, d}^{o}
$$

wherein the matrices $R G_{i}^{*}=R_{i}\left(\eta_{i}^{*}\right) \cdot G_{i}^{T}\left(\rho_{i}^{*}\right)$ and

$$
\begin{aligned}
\Delta R G_{i}= & R_{i}\left(\hat{\eta}_{i}^{*}\right) G_{i}^{T}\left(\hat{\rho}_{i}^{*}\right)+\Delta R_{i}\left(\eta_{i}^{*}, \hat{\eta}_{i}\right) G_{i}^{T}\left(\hat{\rho}_{i}^{*}\right) \\
& +\Delta R_{i}\left(\eta_{i}^{*}, \hat{\eta}_{i}\right) \Delta G_{i}\left(\rho_{i}^{*}, \hat{\rho}_{i}\right)
\end{aligned}
$$

are introduced for notational convenience. In equation (6) the second summand $\Delta R G_{i} \dot{x}_{\mathrm{obj}, d}^{o}$ reflects the error caused by the translational and rotational uncertainties of the grasp point. If estimated and true grasp geometry coincide then

$$
\hat{\rho}_{i}=\rho_{i}^{*}, \hat{\eta}_{i}=\eta_{i}^{*} \Rightarrow \Delta R G_{i}=0
$$


and the error term vanishes. Uncertainties for the grasp point parameters arise from inaccurate grasping of the object, e.g. grasping of the object by visual servo control. Furthermore, a slipping end effector during the manipulation causes the grasp parameters to drift. Due to these uncertainties, the computed end effector trajectory is composed of a component fully compatible with the object motion $x_{i, d}^{*}(t)$ and a part $\Delta x_{i}(t)$ indicating the deviation from the ideal trajectory

$$
x_{i, d}(t)=x_{i, d}^{*}(t)+\underbrace{\int_{0}^{t} T\left(x_{i, d}\right) \Delta R G_{i} \dot{x}_{\mathrm{obj}, d}^{o}(s) \mathrm{d} s}_{\Delta x_{i}(t)} .
$$

with the transformation matrix $T=\left[I_{3}, 0_{3} ; 0_{3}, Q\left(x_{i, d}\right)\right]$ mapping the rigid body twist to the appropriate derivative of the orientation parameterization. In our case $Q$ represents the quaternion propagation formula.

Assume that the $i$-th manipulator performed an inaccurate grasp, i.e. $\Delta R G_{i} \neq 0$, before starting the manipulation task. A desired object motion $\dot{x}_{\text {obj,d }}^{o}$ will generally lead to an increasing trajectory error $\Delta x_{i}$ in (9). Since the value for $\int_{o}^{t} \dot{x}_{\mathrm{obj}, d}^{o}$ correlates with the effectively deployed workspace of the robots, even small values of $\Delta R G_{i}$ will lead to significant errors in the desired trajectory for mobile manipulators.

\section{B. Closed-loop manipulator dynamics}

Each manipulator implements a cascaded control scheme in order to achieve kinematic coordination during the cooperative manipulation task. We choose a velocity-based motion interface in task-space to realize a desired manipulator motion, e.g. a desired object motion as in (5). Advantageously heterogeneous manipulators can be considered as the individual dynamics is compensated by an underlying computed torque control law [12]. This choice enables to treat all manipulators in a common framework regardless of the specific manipulator type. We further assume in this section that each manipulator features an individual mobile platform, i.e. the joint vector of the $i$-th manipulator is represented by

$$
q_{i}=\left(\begin{array}{c}
q_{i, m} \\
q_{i, b}
\end{array}\right) \in \mathbb{R}^{10}
$$

where $q_{i, m} \in \mathbb{R}^{7}$ denotes the joint vector of the anthropomorphic manipulator and $q_{i, b} \in \mathbb{R}^{3}$ is the joint vector of the mobile base. The implications for the dual-arm manipulators will be discussed in Section III. The nonlinear joint space dynamics of the $i$-th manipulator is represented by

$$
\tau_{i}-\tilde{\tau}_{i}=M_{i}\left(q_{i}\right) \ddot{q}_{i}+C_{i}\left(q_{i}, \dot{q}_{i}\right)+g_{i}\left(q_{i}\right),
$$

wherein $q_{i}, \dot{q}_{i}$ and $\ddot{q}_{i}$ denote the vector of the joint position, velocity and acceleration respectively. $\tau_{i} \in \mathbb{R}^{7}$ is the motor joint torque and $\tilde{\tau}_{i} \in \mathbb{R}^{7}$ is a disturbance term due to model uncertainties or unmodeled interaction forces. $M_{i}, C_{i}$ and $g_{i}$ denote the inertia matrix, the Coriolis term and the gravity vector of the manipulator dynamics with appropriate dimensions. We use the computed torque control law

$$
\tau_{\mathrm{i}, \mathrm{ct}}=M_{i}\left(q_{i, r}\right) u(t)+C_{i}\left(q_{i, r}, \dot{q}_{i, r}\right)+g_{i}\left(q_{i, r}\right)
$$

given the desired joint position and velocity $q_{i, r}$ and $\dot{q}_{i, r}$. The new control input $u(t)$ is a combined feed-forward/PD control law in joint space

$$
u(t)=\ddot{q}_{i, r}+K_{i, P}\left(q_{i, r}-q_{i}\right)+K_{i, D}\left(\dot{q}_{i, r}-\dot{q}_{i}\right)
$$

with the desired joint acceleration $\ddot{q}_{i, r}$ and some positivedefinite control gains $K_{i, P}, K_{i, D} \in \mathbb{R}^{10 \times 10}$. The combination of (12) and (13) tracks the desired joint space trajectory, i.e. $q_{i}=q_{i, r}$, if $\tilde{\tau}_{i}=0$. The closed-loop tracking performance with respect to the disturbing joint torque $\tilde{\tau}_{i}$ is

$$
\frac{\Delta q_{i}(s)}{\tilde{\tau}_{i}(s)}=\frac{M_{i}^{-1}}{s^{2}+K_{i, D} s+K_{i, P}}
$$

with $\Delta q_{i}=q_{i, r}-q_{i}$. Choosing $K_{i, P}$ large enough (as commonly done in position-based impedance control schemes) assures small joint space tracking errors, i.e. $q_{i} \approx q_{i, r}$. The particular combination of (12) and (13) facilitates the implementation of a common task-space motion interface irrespective of the individual manipulator type including manipulators without joint torque sensors.

For any manipulator, the desired joint space trajectory $q_{i, r}(t)$ is generated by mapping a reference task-space trajectory $\dot{x}_{i, r}(t)$ via first order differential kinematics into joint space by using the pseudo-inverse $J_{i}^{\dagger}$ in case of redundant manipulators. This step completes the preceding actions to equip each manipulator with a motion interface in task-space. The pseudo-inverse is usually considered as a function only of the joint angles $q_{i}$. In presence of kinematic uncertainties $J_{i}^{\dagger}$ depends on the set of estimated kinematic parameters $\hat{\theta}_{i}$ [7], too. In our case a closed-loop damped least-squares approach is used for the inverse kinematics resolving simultaneously the manipulator's redundancy via

$$
q_{i, r}(t)=q_{i, r}(0)+\int_{0}^{t} J_{i}^{\dagger}\left(q_{i}, \hat{\theta}_{i}\right) \dot{x}_{i, r}(s) \mathrm{d} s .
$$

Splitting the matrix $J_{i}^{\dagger}=J_{i}^{\dagger *}\left(q_{i}, \theta_{i}^{*}\right)+\Delta J_{i}^{\dagger}\left(q_{i}, \theta_{i}^{*}, \hat{\theta}_{i}\right)$ in terms of ideal and estimated kinematic parameters the computed joint space trajectory is decomposed into

$$
q_{i, r}(t)=q_{i, r}^{*}(t)+\underbrace{\int_{0}^{t} \Delta J_{i}^{\dagger} \dot{x}_{i, r}(s) \mathrm{d} s}_{\Delta q_{i}(t)}
$$

The effective trajectory is therefore the sum of an ideal trajectory $q_{i, r}^{*}(t)$ in absence of kinematic uncertainties and a component $\Delta q_{i}(t)$ due to kinematic uncertainties of manipulator and mobile platform. If the estimated and true kinematic parameter vector are equal then

$$
\hat{\theta}_{i}=\theta_{i}^{*} \Rightarrow \Delta J_{i}^{\dagger}=0
$$

and the corresponding error term vanishes. 


\section{Error Analysis}

In order to obtain a complete description of the accumulated error for uncertain grasp geometry and uncertain manipulator kinematics, we will substitute the reference velocity $\dot{x}_{i, r}$ in (15) with the desired velocity $\dot{x}_{i, d}$ from (6). This yields

$$
\begin{aligned}
q_{i, r}(t) & =q_{i, r}(0)+\int_{0}^{t} J_{i}^{\dagger}\left(q_{i}, \hat{\theta}_{i}\right) \dot{x}_{i, r}(s) \mathrm{d} s \\
& =q_{i, r}(0)+\int_{0}^{t} J_{i}^{\dagger^{*}} R G_{i}^{*} \dot{x}_{\mathrm{obj}, d}^{o} \mathrm{~d} s \\
& +\int_{0}^{t}\left(\Delta J_{i}^{\dagger}\left(R G_{i}^{*}+\Delta R G_{i}\right)+J_{i}^{\dagger *} \Delta R G_{i}\right) \dot{x}_{\mathrm{obj}, d}^{o} \mathrm{~d} s .
\end{aligned}
$$

The very last term in (19) expresses the error of the accumulated error in joint space. We derive further from (19) that several factors influence the deviation of the end effector trajectory. Fast and persistent motion, increased uncertainty in the grasp geometry and longer manipulation time lead to higher deviations of the end effector trajectory. Also the actual manipulator configuration has an impact on the error term in equation (19) which will not be discussed here in detail due to space constraints. Subsequently we illustrate the impact of the accumulated kinematic errors on the interaction forces.

\section{Interaction forces}

The interaction force model serves to formally illustrate the impact of kinematic uncertainties as described in the preceding subsection. A rigid grasp is assumed for the end effectors involved in the manipulation task. Thus the relative pose of the end effectors is constrained through the object, i.e.

$$
\left\|x_{j}-x_{i}\right\|=\text { const. }
$$

for all $i \neq j$ and $\forall t>0$. This constraint will always be enforced by an appropriate value of the emerging end effector forces. While the detailed force control scheme for the manipulators is presented in Section III-A, it is convenient to focus on the effect of uncertain kinematic parameters. The relative displacement between the $i$-th and the $j$-th end effector due to the desired velocities is computed according to

$$
\Delta x_{j i, d}(t)=\int_{0}^{t} T\left(\Delta x_{j i, d}\right)\left[\dot{x}_{j, d}(s)-\dot{x}_{i, d}(s)\right] \mathrm{d} s
$$

which potentially violates the constraint. Whenever the desired relative displacement $\Delta x_{j i, d}^{o}(t)$ does not meet the constraint in (20), undesired end effector forces emerge which enforce this constraint to be met. This is usually the case for a bias in the kinematic parameters leading to desired positions violating the constraint. The induced force is commonly known as internal force and can be approximated by

$$
f_{j i, \text { int }}^{o}(t)=-f_{i j, \text { int }}^{o}(t) \approx K_{j i} \Delta x_{j i, d}^{o}(t)
$$

for $i \neq j$. The induced force $f_{j i \text { int }}^{o}(t)$ is proportional to the effective stiffness $K_{j i}=K_{i j}>0$ along the connecting line of the manipulators $i$ and $j$ which can be tuned by means of the individual end effector stiffnesses. For a manipulation scenario involving $N>1$ manipulators the end effector force will be the superposition of all forces due to relative displacements of the end effector ensemble. An illustration of this model is depicted in Fig. 3.

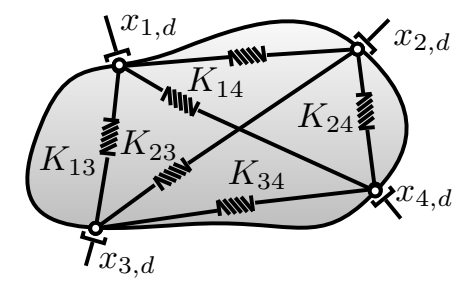

Fig. 3. Illustration of the interaction force model for $N=4$

Note that the desired pose of the $i$-th manipulator $x_{i, d}$ represents the (virtual) suspension point for the manipulator's impedance control scheme while the reference pose $x_{i, r}$ is the effectively tracked manipulator pose. The interaction structure equals a complete graph, since kinematic errors of one manipulator will be propagated to any other manipulator in terms of internal forces [13]. The analysis of internal forces is briefly reviewed below.

Given the measured end effector forces $f_{i}^{o} \in \mathbb{R}^{6}$, the net force about the object's center of mass $f_{\text {obj }}^{o} \in \mathbb{R}^{6}$ is a function of all applied end effector forces computed via

$$
f_{\mathrm{obj}}^{o}=\left[G_{1} \cdots G_{N}\right] f^{o}=G f^{o}
$$

where $f^{o}=\left[\left(f_{1}^{o}\right)^{T} \cdots\left(f_{N}^{o}\right)^{T}\right]^{T} \in \mathbb{R}^{6 N}$ is the stacked vector of the force/torque measurements expressed in the object frame. $G$ is called the grasp matrix. The force components inducing no effective motion of the object lie in the null space of the grasp matrix $G$ and are computed via

$$
f_{\text {int }}^{o}=\left(I-G^{\dagger} G\right) f^{o}
$$

wherein $G^{\dagger}$ is a particular ("no-squeeze") pseudoinverse [14] of $G$ according to

$$
G^{\dagger}=\frac{1}{N}\left[\begin{array}{ccccc}
I_{3} & S\left(\hat{\rho}_{1}\right) & \ldots & I_{3} & S\left(\hat{\rho}_{N}\right) \\
0_{3} & I_{3} & \ldots & 0_{3} & I_{3}
\end{array}\right]^{T} .
$$

Note that $f_{\text {int }}^{o} \in \mathbb{R}^{6 N}$ contains the end effector force/torque components contributing to internal stress on the object. Similarly, the end effector force/torque contributing to a net motion of the object is $f_{\text {ext }}^{o} \in \mathbb{R}^{6 N}$ computed by means of

$$
f_{\mathrm{ext}}^{o}=G^{\dagger} G f^{o} .
$$

Employing (23) one can show that any superposition of the force components defined in (22) belong actually to the space of internal forces since both vectors have same magnitude but opposed signs. 


\section{Control Architecture}

\section{A. Manipulator control}

The desired object dynamics are usually specified in terms of an impedance [2] with tunable parameters $M_{o}, D_{o}$ and $K_{o}$ representing the mass, damping and stiffness matrix respectively. The deviation from the desired trajectory $\Delta x_{\mathrm{imp}}^{o}$ due to an external force on the object $f_{\text {obj }}^{o}$ is computed via

$$
M_{o} \Delta \ddot{x}_{\mathrm{imp}}^{o}+D_{o} \Delta \dot{x}_{\mathrm{imp}}^{o}+K_{o} \Delta x_{\mathrm{imp}}^{o}=f_{\mathrm{obj}}^{o} .
$$

The desired object motion $\Delta \dot{x}_{\text {imp }}^{o}$ due to the external force requires a centralized evaluation of all current end effector forces as indicated in (23). We bypass this inconvenience by following the approach presented in [3] and distribute the desired object impedance to corresponding manipulator impedances. The effective motion of the $i$-th manipulator $\Delta \dot{x}_{\mathrm{i}, \mathrm{imp}}$ due to force impact is computed locally by

$$
M_{i} \Delta \ddot{x}_{\mathrm{i}, \mathrm{imp}}+D_{i} \Delta \dot{x}_{\mathrm{i}, \mathrm{imp}}+K_{i} \Delta x_{\mathrm{i}, \mathrm{imp}}=\Delta f_{i},
$$

where $\Delta f_{i}=f_{i}-f_{i, g}$ is the gravity compensated end effector force. The compensation term $f_{i, g}$ represents an external end effector force due to the object mass $m_{o}$ computed by letting $G f^{o}=\left[-m_{o} g^{T}, 0,0,0\right]^{T}$ in (26). $M_{i}, D_{i}$ and $K_{i}$ are the mass, damping and stiffness matrix of the manipulator impedance. Equivalent object impedance parameters $M_{o}, D_{o}$ and $K_{o}$ can be computed and tuned by means of $M_{i}, D_{i}$, $K_{i}$ and a given grasp geometry.

\section{B. Mobile platform control}

Up to this point the mobile platform is represented in terms of additional degrees of freedom $q_{i, b}$ for each individual manipulator of the model in (11). In this section we assume that the manipulators $i$ and $j$ share a common mobile platform $k$ and build thus a dual-arm system. The first order differential kinematics of the dual-arm manipulator can be written as

$$
\left[\begin{array}{c}
\dot{x}_{i} \\
\dot{x}_{j}
\end{array}\right]=\underbrace{\left[\begin{array}{ccc}
J_{i, m}^{*}\left(q_{i, m}\right) & 0 & J_{k, b}^{*}\left(q_{k, b}\right) \\
0 & J_{j, m}^{*}\left(q_{j, m}\right) & J_{k, b}^{*}\left(q_{k, b}\right)
\end{array}\right]}_{J_{k}\left(q_{i, m}, q_{j, m}, q_{k, b}\right)}\left[\begin{array}{c}
\dot{q}_{i, m} \\
\dot{q}_{j, m} \\
\dot{q}_{i=j, b}
\end{array}\right]
$$

with $J_{i, m}^{*}, J_{j, m}^{*} \in \mathbb{R}^{6 \times 7}$ and $J_{k, b}^{*} \in \mathbb{R}^{3 \times 3}$ denoting the kinematically unbiased Jacobian of the $i$-th and $j$-th manipulator and the Jacobian of the $k$-th mobile platform respectively. Calculation of the pseudo-inverse of $J_{k}$ in equation (29) requires in general complex computations since $J_{k} J_{k}^{T} \in \mathbb{R}^{12 \times 12}$ is generally not sparse. For our particular case the mobile platform Jacobian is the identity matrix, i.e. $J_{k, b}^{*}=I_{3}$. This is easily verified by considering the Cartesian pose of the $k$-th mobile platform $x_{k, b}$ which coincides with the joint angles of the $i$-th and $j$-th manipulator

$$
x_{k, b}=\left(\begin{array}{ccc}
p_{k, x} & p_{k, y} & \varphi_{k, z}
\end{array}\right)^{T}=q_{i, b}=q_{j, b}
$$

wherein $p_{k, x}, p_{k, y} \in \mathbb{R}$ denote the mobile platform position in $\mathrm{x}$-y-plane and $\varphi_{k, z} \in \mathbb{R}$ the current orientation in this plane. We propose to decouple the manipulator motion and the mobile platform motion kinematically in task-space which appears favorable in view of the following considerations:

- Disturbances of the mobile platform acting on the signal $\dot{q}_{i=j, b}$ such as delayed or imprecise wheel actuation propagate through the coupled kinematics immediately to the end effectors of both manipulators (cf. (29)). When decoupling the mobile platform from the manipulators, disturbances are efficiently compensated before propagating to end effector level. No additional requirements need to be laid down for the null-space dynamics of the kinematic chain built by manipulators and mobile base.

- The pseudo-inverse $J_{k}^{+}$does not need to be computed which reduces the computational complexity significantly and deploys the pseudo-inverses of the individual manipulators $J_{i, m}^{*+}$ and $J_{j, m}^{*+}$ for the implementation. These are of lower dimension than $J_{k, b}^{*}$ and are commonly already available for a specific manipulator.

To achieve the desired decoupling, we adopt an approach similar to [4] but formulate the employed potential function in task-space. The desired relative platform position with respect to the end effector poses $\left(x_{i}\right.$ and $\left.x_{j}\right)$ is expressed in terms of the potential function $V_{\text {base }}\left(x_{k, b}, x_{i}, x_{j}\right)$. This potential is chosen to have a minimum at the desired translational and rotational offset $\left[d_{x}, d_{y}, d_{\varphi}\right]$. To this end we extract the geometric center of the end effectors $i$ and $j$ in the $\mathrm{x}$-y-plane from $p_{c}=\frac{x_{i}+x_{j}}{2}$ and their relative distance from $\Delta p=\frac{x_{i}-x_{j}}{2}$. The introduced variables are illustrated in Fig. 4.

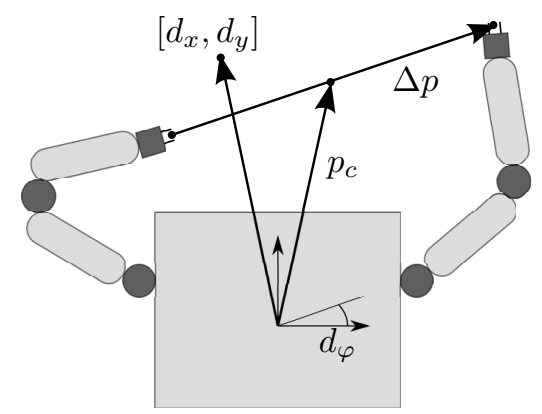

Fig. 4. Illustration of the desired platform offset

The proposed potential function reads as

$$
\begin{aligned}
V_{\text {base }}\left(x_{k, b}\right)= & c_{x}\left[\left(p_{c, x}-p_{k, x}\right)-d_{x}\right]^{2} \\
& +c_{y}\left[\left(p_{c, y}-p_{k, y}\right)-d_{y}\right]^{2} \\
& +c_{\varphi}\left[\left(\arctan \frac{\Delta p_{x}}{\Delta p_{y}}-\varphi_{k, z}\right)-d_{\varphi}\right]^{2}
\end{aligned}
$$

wherein the additional subscripts denote the $x$ - and $y$ components of the respective vector expressed in the current base frame. Choosing (w.l.o.g.) the manipulator's base frame 
to be aligned with the frame of the mobile platform, the position $p_{k}$ and the orientation $\varphi_{k}$ of the mobile platform w.r.t. the base frame is constant and possibly zero, i.e. $p_{k}=0$ and $\varphi_{k, z}=0$. The coefficients $c_{x}, c_{y}, c_{\varphi} \in \mathbb{R}^{+}$are positive control gains. Subsequently a gradient descent towards the minimum of the potential function (31) is performed which defines the desired platform motion

$$
\dot{x}_{k, b}=-\nabla V_{\text {base }}\left(x_{k, b}\right) .
$$

In order to achieve the desired decoupling, the induced platform motion is compensated by adding the converse of the computed platform velocity signal to the desired end effector trajectories $\dot{x}_{i, d}$ and $\dot{x}_{j, d}$ in terms of

$$
\Delta \dot{x}_{i, b}=\Delta \dot{x}_{j, b}=+\nabla V_{\text {base }}\left(x_{k, b}\right)
$$

This procedure allows to steer the mobile platform hierarchically by performing desired end effector trajectories and dragging the platform to minimize the criterion in equation (31).

\section{Overall Control Architecture}

Combining the results from the previous sections, the reference velocity for the $i$-th end effector is

$$
\dot{x}_{i, r}=\dot{x}_{i, d}+\Delta \dot{x}_{i, \text { imp }}+\Delta \dot{x}_{i, b} .
$$

The reference signal $\dot{x}_{i, r}$ incorporates a feed-forward term $\dot{x}_{i, d}$ due to the desired object velocity, a compliance term $\Delta \dot{x}_{i \text { imp }}$ realizing the desired impedance behavior and a term $\Delta \dot{x}_{i, b}$ providing the coordination of manipulator and mobile platform in the end effector null-space: by letting $\dot{q}_{i=j, b}=+\nabla V_{\text {base }}$ with some proper inverse kinematics such that $J_{i, m}^{*} \dot{q}_{i, m}=J_{j, m}^{*} \dot{q}_{j, m}=-\nabla V_{\text {base }}$ it follows immediately from (29) that $\dot{x}_{i}=\dot{x}_{j}=0$. Thus (34) implements a distributed impedance scheme with local trajectory tracking while decoupling the end effector motion from potential mobile base disturbances. The resulting control architecture is depicted exemplarily for two manipulators mounted on the same mobile platform in Fig. 5.

The desired object-velocity $\dot{x}_{\mathrm{obj}, d}^{o}$ is transformed by means of (5) to desired end effector velocities $\dot{x}_{i, d}$. While tracking the computed end effector trajectory, the measured end effector forces are employed for evaluating the distributed impedance equations (28). Varying end effector positions will induce a platform motion in order to minimize the potential function defined in (31).

The kinematic approximation in (34) for disturbance decoupling implies that dynamical effects relevant to the platform dynamics are neglected. Dynamical effects due to non-compensated wheel inertia or increasing wheel torques for an augmented payload enter the control loop as additional disturbances. Their impact remains small for low and smooth accelerations of the manipulator system.

\section{EXPERIMENTAL VALIDATION}

\section{A. Experimental setup}

The first of two robots used for the experimental evaluation consists of two 7DoF robotic manipulators [15] with incremental encoders capturing the joint angles. Due to the incremental encoders in use this manipulator type is prone to kinematic uncertainties. The second robot is equipped with two commercially available KUKA LWR 4+ manipulators [16]. Both dual-arm manipulators are front-mounted on top of a rigid torso as depicted in Fig. 1.

The workspace of the robotic manipulators is extended by a four-wheeled omni-directional mobile platform [17]. A JR3 $6 \mathrm{DoF}$ force/torque sensor is mounted at the wrist of each manipulator. For a tight grasp of the object every manipulator features a Schunk $P G 70$ parallel gripper with two metal fingers matching the object.

The low-level control algorithms are implemented by means of MATLAB/Simulink Real-Time Target models running with a sampling time of $T_{s}=1 \mathrm{~ms}$. Coordination is achieved by appropriate interfaces to a high-level software control framework [18]. For inter-robot data exchange a wireless router transmitting over the UDP protocol is used. Packet loss and time delay were negligible during the experiment.

\section{B. Experimental results}

The manipulated object consists of a reinforced aluminum frame with handholds for the robots. The rigid frame of length $l_{0}=1.20 \mathrm{~m}$ and width $w_{0}=0.80 \mathrm{~m}$ has a total weight of $m_{0}=3.2 \mathrm{~kg}$. The grasp geometry $\hat{\rho}_{i}, \hat{\eta}_{i}$ is known to each manipulator during the task execution. For the manipulation task, the impedance control parameters for $i=\{1, \ldots, 4\}$ in (28) are set to $M_{i}=\left[6 I_{3} \mathrm{~kg}, 0_{3} ; 0_{3}, 0.5 I_{3} \mathrm{kgm}^{2}\right]$, $D_{i}=\left[200 I_{3} \mathrm{Ns} / \mathrm{m}, 0_{3} ; 0_{3}, 10 I_{3} \mathrm{Nms} / \mathrm{rad}\right] \quad$ and $K_{i}=\left[200 I_{3} \mathrm{~N} / \mathrm{m}, 0_{3} ; 0_{3}, 10 I_{3} \mathrm{~N} / \mathrm{m}\right] . \quad$ The parameters for the mobile base coordination in (31) are set to $d_{x}=0.80 \mathrm{~m}, d_{x}=0.0 \mathrm{~m}$ and $d_{\varphi}=0 \mathrm{rad}$. The chosen object trajectory is a pure translation in the $x-y-p l a n e$, starting at the origin and describing an eight as illustrated by the black dashed line in Fig. 6.

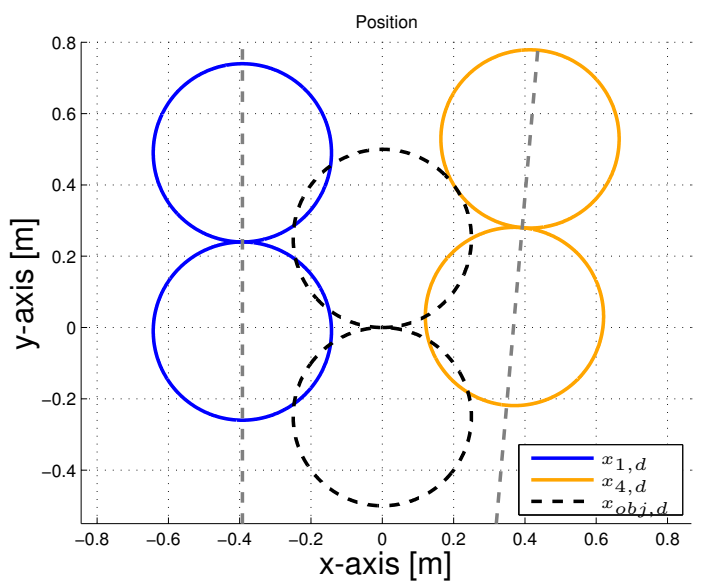

Fig. 6. Desired object trajectory and biased end effector trajectory $x_{4, d}$ 


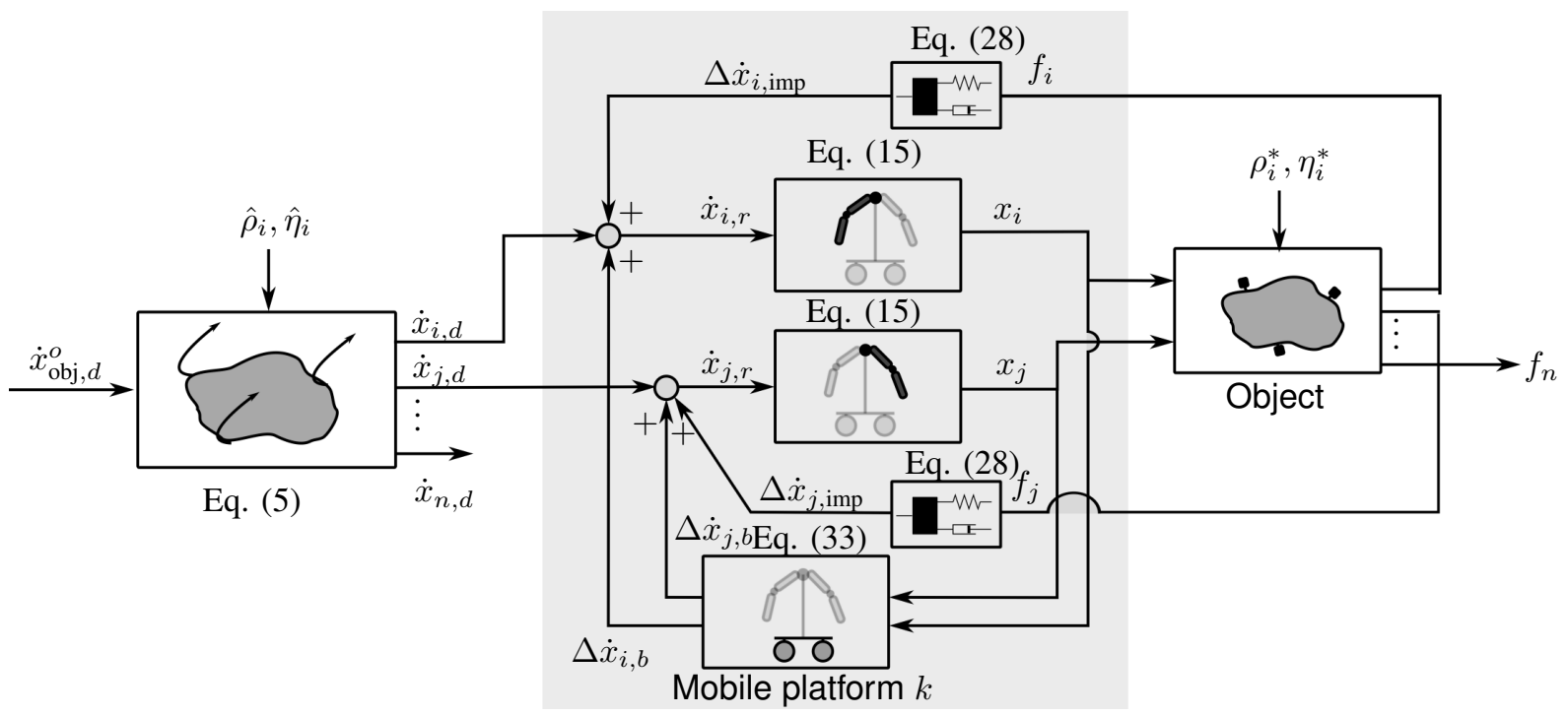

Fig. 5. Schematic overview of the cooperative mobile manipulation control architecture

Each circle has a diameter of $d=0.50 \mathrm{~m}$ and is tracked within $T=20 \mathrm{~s}$. This leads to a maximum end effector velocity of $v_{\max }=\frac{d}{2} \frac{2 \pi}{T}=0.078 \frac{\mathrm{m}}{\mathrm{s}}$ and a task duration of $2 T=40 s$. By choosing sufficiently small velocities the impact of dynamic forces is eliminated during the transportation phase permitting to focus on the kinematic decoupling of manipulator and mobile base. The maximum displacement of $1.0 \mathrm{~m}$ for the given trajectory is sufficient to illustrate the benefits of the control architecture and the effects relevant to uncertain kinematic parameters. The grasp points of the four involved end effectors are identified using a ruler: $\hat{\rho}_{1}^{o}=[0.39,-0.24,0]^{T} \mathrm{~m}, \quad \hat{\rho}_{2}^{o}=[0.39,0.24,0]^{T} \mathrm{~m}$, $\hat{\rho}_{3}^{o}=[0.39,-0.28,0]^{T} \mathrm{~m} \quad$ and $\quad \hat{\rho}_{4}^{o}=[-0.39,0.28,0]^{T} \mathrm{~m}$. The estimated orientation of the end effectors is $\hat{\eta}_{1}^{o}=\hat{\eta}_{2}^{o}=[0,0,0,1]^{T}$ and $\hat{\eta}_{3}^{o}=\hat{\eta}_{4}^{o}=[1,0,0,0]^{T}$ given as unit quaternions with the first entry denoting the scalar component. The end effector force signals are recorded and the internal force component is extracted according to (24). The captured force signals are filtered by a moving average filter with a window size of $T_{m a}=300 \mathrm{~ms}$. For the purpose of a clear presentation only the force signals of the opposed end effectors $i=\{1,4\}$ are plotted in Fig. 7.

While performing the manipulation task the decoupling of manipulators and mobile platform is considered satisfactory with a remaining standard deviation of the end effector forces of $\sigma\left(f_{1}\right)=2.16 \mathrm{~N}$ and $\sigma\left(f_{4}\right)=1.34 \mathrm{~N}$. The applied stress on the object remains small. However we still observe the impact of a non-ideal platform steering. At time instants $t=$ $15 \mathrm{~s}$ and $t=36 \mathrm{~s}$ we observe peaks in the force signals which arise from the non-compensated wheel-turning action at the reversal points of the trajectory. The remaining fluctuations of the force measurements apparent in both end effector signals are attributed to the interaction of the four impedancecontrolled end effectors. The distinct offset of $f_{4 \text {,int }}^{o}$ is caused by an initial tension when closing the gripper jaw.

In a second run, the estimated grasp orientation of

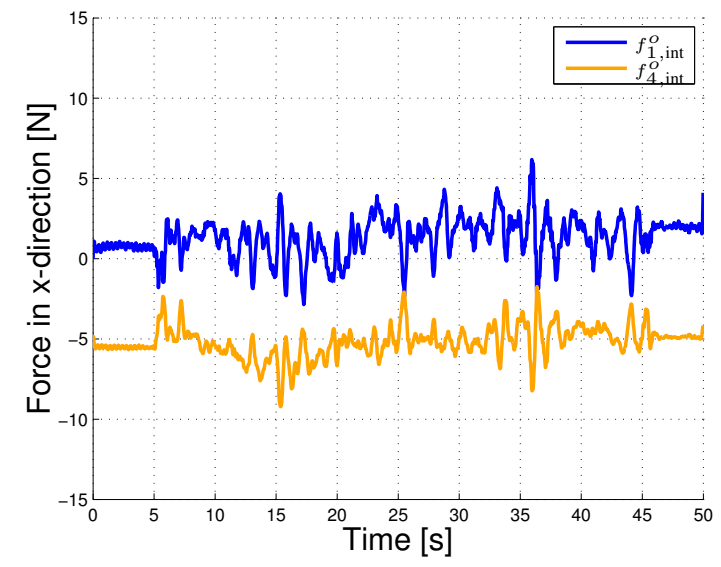

Fig. 7. Internal force for unbiased grasp parameters

the fourth gripper is intentionally biased by a rotation of 5 degrees about the $\mathrm{z}$-axis. This value is considered as a worst-case estimation of the kinematic uncertainty when grasping the object autonomously with a local visual servo control routine. The resulting grasp orientation is therefore $\hat{\eta}_{4, \text { biased }}^{o}=[0.999,0,0,0.0436]^{T}$. This choice will generate a biased trajectory for the fourth end effector with respect to the desired object trajectory as depicted by the yellow line in Fig. 6. During the upper loop of the trajectory, the biased end effector moves about $2 \mathrm{~cm}$ too far in $\mathrm{x}$-direction due to the orientation offset. Considering the model of the interaction force (22) due to relative end effector displacement, one expects the biased end effector to induce stress in negative $x$-direction during the upper loop and in positive $\mathrm{x}$-direction during the lower loop. The end effector force signals for $i=\{1,4\}$ and a biased end effector trajectory are plotted in Fig. 8.

The force signal $f_{4 \text {,int }}^{o}$ of the biased end effector shows the amplitude as expected. Again we notice some force 


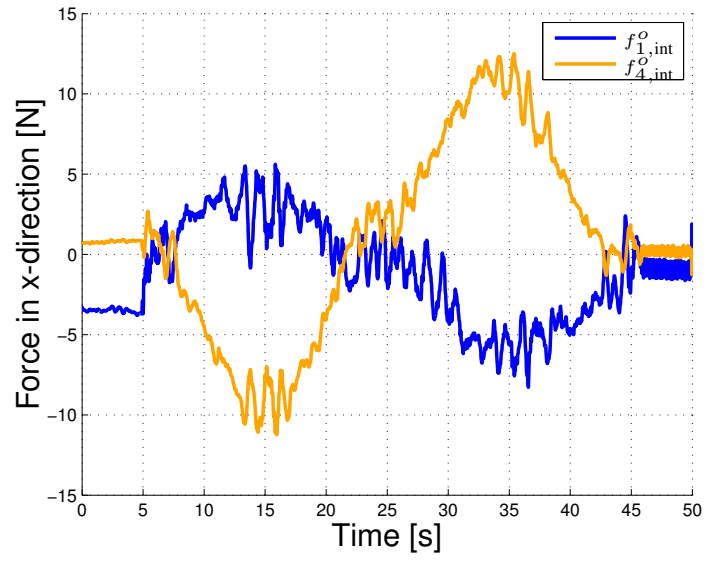

Fig. 8. Internal force for biased grasp parameters

peaks at the reversal points of the trajectory due to the nonideal platform motion. The remaining force signals $f_{i, \text { int }}^{o}$ for $i=\{1,2,3\}$ share the applied stress with converse signs. For non-periodic manipulation trajectories the observed decrease of the internal stress does not apply. Instead the error induced by uncertain kinematics will build up, induce further stress on the object and limit thus the actually usable workspace of the cooperative manipulation system.

The presented results build the basis for deriving a tuning strategy for the individual impedance parameters since different choices for $K_{i}$ may realize the same desired object stiffness $K_{o}$ in (27). The parameter tuning is not part of this paper and will be investigated in future work. An additional measure to extend the usable workspace is the use of an internal force controller. However, since the biased kinematic parameters enter the system in the feed-forward path, asymptotic tracking of force and position trajectories will rely on augmented controller dynamics for specific reference signal types. The design of an adaptive control scheme to overcome this problem is introduced in a companion paper [9].

\section{CONCLUSION}

In this paper we present a control scheme for cooperative, multi-robot dual-arm mobile manipulators. Our approach extends a distributed impedance control scheme for efficient coordination of the mobile manipulators. We propose a strategy to achieve decoupling of the kinematic tree built by the manipulators and the mobile base in task-space. We systematically derive the impact of kinematic uncertainties arising at object and manipulator level and identify them as a major challenge in cooperative mobile manipulation. The presented scheme is applicable in real-time and allows to integrate heterogeneous manipulator types for the execution of the manipulation task. An experimental study conducted with two dual-arm mobile manipulators completes our work and validates the efficacy of the proposed approach. The study of dynamic decoupling strategies, a rigorous stability analysis and the control tuning is part of the future work.

\section{ACKNOWLEDGMENTS}

This research is partly supported by the DFG excellence initiative research cluster "CoTeSys - Cognition for Technical Systems" and the European Union Seventh Framework Programme FP7/20072013 under grant agreement no. 601165 of the project "WEARHAP - Wearable Haptics for Humans and Robots“.

\section{REFERENCES}

[1] N. Hogan, "Impedance control: An approach to manipulation: Part I - Theory," Journal of Dynamic Systems, Measurement, and Control, vol. 107, no. 1, pp. 1-7, 1985.

[2] S. Schneider and R. Cannon, "Object Impedance Control for Cooperative Manipulation: Theory and Experimental Results," IEEE Trans. Robot. Automat., vol. 8, no. 3, pp. 383-394, 1992.

[3] J. Szewczyk, F. Plumet, and P. Bidaud, "Planning and controlling cooperating robots through distributed impedance," Journal of Robotic Systems, vol. 19, no. 6, pp. 283-297, 2002.

[4] O. Khatib, K. Yokoi, K. Chang, and D. Ruspini, "Force strategies for cooperative tasks in multiple mobile manipulation systems," ROBOTICS, 1996.

[5] T. Sugar and V. Kumar, "Control of cooperating mobile manipulators," Robotics and Automation, IEEE Transactions on, vol. 18, no. 1, pp 94-103, Feb.

[6] K. Kosuge, H. Kakuya, and Y. Hirata, "Control algorithm of dual arms mobile robot for cooperative works with human," in Systems, Man, and Cybernetics, 2001 IEEE International Conference on, vol. 5, pp. 3223-3228 vol.5.

[7] C. Cheah, C. Liu, and J. Slotine, "Adaptive jacobian tracking control of robots with uncertainties in kinematic, dynamic and actuator models," Automatic Control, IEEE Transactions on, vol. 51, no. 6, pp. 1024 1029, june 2006.

[8] F. Aghili, "Self-tuning cooperative control of manipulators with position/orientation uncertainties in the closed-kinematic loop," in Intelligent Robots and Systems (IROS), 2011 IEEE/RSJ International Conference on, Sept. 2011, pp. $4187-4193$.

[9] S. Erhart and S. Hirche, "Adaptive force/velocity control for multirobot cooperative manipulation under uncertain kinematic parameters,' in Intelligent Robots and Systems (IROS), 2013 IEEE/RSJ International Conference on, Nov. 2013.

[10] Y. Kume, Y. Hirata, Z. dong Wang, and K. Kosuge, "Decentralized control of multiple mobile manipulators handling a single object in coordination," in Intelligent Robots and Systems, 2002. IEEE/RSJ International Conference on, vol. 3, pp. 2758-2763 vol.3.

[11] B. Siciliano and O. Khatib, Springer Handbook of Robotics. Secaucus, NJ, USA: Springer-Verlag New York, Inc., 2007.

[12] B. Siciliano, L. Sciavicco, L. Villani, and G. Oriolo, Robotics: Modelling, Planning and Control, 1st ed. Springer Publishing Company, Incorporated, 2008.

[13] D. Williams and O. Khatib, "The virtual linkage: a model for internal forces in multi-grasp manipulation," in Robotics and Automation, 1993. Proceedings., 1993 IEEE International Conference on, 1993, pp. $1025-1030$ vol.1.

[14] I. D. Walker, R. A. Freeman, and S. I. Marcus, "Analysis of motion and internal loading of objects grasped by multiple cooperating manipulators," The International Journal of Robotics Research, vol. 10 , no. 4, pp. 396-409, 1991.

[15] B. Stanczyk and M. Buss, "Development of a Telerobotic System for Exploration of Hazardous Environments," in Proc. IEEE/RSJ IROS, 2004, pp. 2532-2537.

[16] R. Bischoff, J. Kurth, G. Schreiber, R. Koeppe, A. Albu-Schaeffer, A. Beyer, O. Eiberger, S. Haddadin, A. Stemmer, G. Grunwald, and G. Hirzinger, "The KUKA-DLR Lightweight Robot arm - a new reference platform for robotics research and manufacturing," in Robotics (ISR), 201041 st International Symposium on and 2010 6th German Conference on Robotics (ROBOTIK), June, pp. 1-8.

[17] U. Hanebeck, N. Saldic, and G. Schmidt, "A Modular Wheel System for Mobile Robot Applications," in Proc. IEEE/RSJ IROS, 1999, pp. $17-22$.

[18] M. Rambow, F. Rohrmüller, O. Kourakos, D. Bršcic, D. Wollherr, S. Hirche, and M. Buss, "A Framework for Information Distribution, Task Execution and Decision Making in Multi-Robot Systems," IEICE transactions on information and systems, vol. 93, no. 6, pp. 13521360,2010 